\title{
A Computer-Controlled Tensile Stage for In-Situ X-Ray Measurements
}

\section{E. Levine}

School of Mechanical and Materials Engineering Washington State University

Pullman, Washington 99164

\section{R. J. Fields}

Metallurgy Division

U.S. DEPARTMENT OF COMMERCE

Technology Administration

National Institute of Standards

and Technology

Gaithersburg, MD 20899-0001

QC

100

.U56

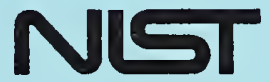

NO.5867 



\section{NISTIR 5867}

\section{A Computer-Controlled Tensile Stage for In-Situ X-Ray Measurements}

\section{E. Levine}

School of Mechanical and Materials Engineering Washington State University

Pullman, Washington 99164

\section{R. J. Fields}

Metallurgy Division

U.S. DEPARTMENT OF COMMERCE

Technology Administration

National Institute of Standards

and Technology

Gaithersburg, MD 20899-0001

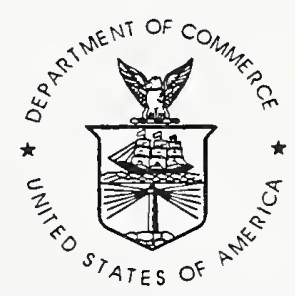

U.S. DEPARTMENT OF COMMERCE

William M. Daley, Secretary

TECHNOLOGY ADMINISTRATION

Gary R. Bachula, Acting Under Secretary for Technology

NATIONAL INSTITUTE OF STANDARDS

AND TECHNOLOGY

Raymond G. Kammer, Director 



\title{
A computer-controlled tensile stage for in situ X-ray measurements
}

\author{
L. E. Levine \\ School of Mechanical and Materials Engineering \\ Washington State University \\ Pullman, WA \\ R. J. Fields ${ }^{1}$ \\ National Institute of Standards and Technology \\ Gaithersburg, $M D$
}

(November 18, 1996)

\begin{abstract}
Plastic deformation in metals results in dramatic changes in their mechanical properties; such changes are caused by the evolution of complicated, threedimensional networks of dislocations. Conducting in situ X-ray studies of this bulk process requires that samples be strained while they are mounted on an $\mathrm{X}$-ray goniometer. In order to compare the developing dislocation structures with the changing mechanical properties, stress-strain measurements must be made simultaneously with the X-ray studies. A low-profile, computercontrolled tensile stage has been developed that is both small and light enough to mount on an X-ray goniometer; it can strain a sample in situ and can measure both the average longitudinal stress and the average linear strain.
\end{abstract}

\section{INTRODUCTION}

The changes in the bulk mechanical properties of plastically deformed metals are caused by the evolution of complicated dislocation structures that take the form of cells at large strains. Studying the development of such structures in single-crystal metal samples deformed uniaxially in tension is a logical first step in understanding the more general problem. Even for this relatively simple case, the dislocation pattern formation is very sensitive to such parameters as the orientation of the strain axis, sample temperature, strain rate and the starting quality of the sample. Clearly, it is necessary to conduct experiments that can examine the dislocation structures in situ while simultaneously measuring the stress and strain of the sample.

Since the formation of dislocation cells is a bulk process and since these cells usually extend over several micrometers in size, a minimum sample thickness of approximately 100 $\mu \mathrm{m}$ is required for in situ experiments. This eliminates the use of electrons as a possible 
probe leaving X-rays and neutrons as the most viable candidates. The high brightness and small source size of X-ray synchrotron radiation, along with the high spatial resolution and sensitivity of X-ray detectors, makes X-rays the preferred probe for these in situ measurements.

A combination of several complementary X-ray techniques is required to fully characterize the dislocation structure evolution. The early stages of the deformation process can be studied using monochromatic, high-resolution diffraction imaging on single-crystal samples deformed in situ. These experiments are being conducted on beam line X23A3 of the National Synchrotron Light Source (NSLS) at Brookhaven National Laboratory. This technique allows in situ microscopic, stereoscopic imaging of the dislocations as the sample is strained. ${ }^{2,3}$ Later stages of the deformation process are being studied in situ using small angle X-ray scattering (SAXS) and line profiling of X-ray Bragg peaks. ${ }^{4-6}$

To conduct these in situ X-ray experiments, we have designed and built a computercontrolled low-profile tensile stage that is both small and light enough to be mounted on an X-ray goniometer. It can deform a specimen in situ while simultaneously measuring the average longitudinal stress and the average linear strain. To avoid introducing undesirable defects into the sample, the tensile stage was carefully designed to apply tension along a well defined axis with minimal torques. Measurements of dislocation structures during monotonic deformation, fatigue, creep, and relaxation are all possible using this equipment. Although the tensile stage system was designed for the dislocation studies described above, the stage can be used for any in situ X-ray experiments where an applied axial stress is required.

\section{DESCRIPTION OF TENSILE STAGE SYSTEM}

As shown in the block diagram in Fig. 1, the tensile stage system has four main sections. Section one is the tensile stage which includes the sample clamping mechanism, a miniature stepping motor with a gear reduction of about 500:1, a worm-drive, four capacitance displacement sensors, and a limit switch. The tensile stage is the only part of the system that is mounted on the goniometer. The frame of the tensile stage has dimensions $101.6 \mathrm{~mm} \times$ $69.85 \mathrm{~mm} \times 12.7 \mathrm{~mm}\left(4 \mathrm{in} . \times 2 \frac{3}{4} \mathrm{in} . \times \frac{1}{2}\right.$ in.). To save weight, most of the stage is built of an aluminum alloy; the weight of the complete tensile stage is just $0.45 \mathrm{~kg}$.

The second section is the tensile stage controller which runs the stepping motor and monitors the limit switch. The controller has a built-in programmable microprocessor that is operated remotely via an RS 422 interface.

The third section is a rack of amplifiers for the capacitance sensors. These amplifiers are connected to the sensors with miniature BNC cables; the amplifiers generate a voltage proportional to the displacement in the range 0 to $10 \mathrm{~V}$, with a rated resolution of $1 \mathrm{mV}$.

The final section of the tensile stage system is an 80386-based computer that both controls the operation of the tensile stage and logs the data from the capacitance sensors. The capacitance data is processed and displayed in real time as values of the average linear strain and sample rotation (typically $\ll 0.1^{\circ}$ when the sample is properly clamped). The computer is equipped with a 16 bit A/D board that samples the output of the capacitance sensor amplifiers; commercially available software is used to control the A/D board. Displacement data are taken at $0.5 \mathrm{~s}$ intervals and stored on the hard drive. A simple multitasking terminal 
program is sufficient to control and program the stepping motor via the tensile stage control box. The computer is the only part of the system that is situated outside of the X-ray shielded hutch.

Figure 2 shows a detailed drawing of the tensile stage. The stepping motor has a maximum torque of $\approx 0.07 \mathrm{Nm}$ and a step angle of $6^{\circ}$. A built-in reduction gear box provides a reduction ratio of $128: 1$ and limits the maximum rotation speed of the stepping motor to $4000 \mathrm{rpm}$. A small spur gear attached to the output spindle of the gear box turns the main spur gear with a resulting reduction ratio of 4:1. The total reduction ratio is therefore 512:1.

The center of the main gear has a $9.525 \mathrm{~mm}\left(\frac{3}{8} \mathrm{in}\right.$.) bore that is tapped at $0.945 \mathrm{turns} / \mathrm{mm}$ (24 turns/in.). As the gear rotates, it translates a threaded shaft that provides the required axial displacement. The shaft is machined to have a square cross-section where it passes through a square hole in the rectangular frame of the tensile stage. This geometry prevents the shaft from rotating with the main gear. Since the axial force on the main gear can be considerable, it rests on a low-friction thrust bearing rated at $890 \mathrm{~N}$. With the existing gearing, this is the limiting force that the motor can apply to the sample.

Taking all gear reductions into account and ignoring compliance in the system, a single $6^{\circ}$ step of the stepping motor produces a shaft translation of just $3 \times 10^{-2} \mu \mathrm{m}$. Since the stepping motor controller allows micro-stepping of the motor, the sample deformation is effectively continuous even at extremely small strain rates. Conversely, running the stepping motor at its maximum allowed speed of $4000 \mathrm{rpm}$ produces a maximum pull rate of $130 \mu \mathrm{m} / \mathrm{sec}$. Samples are generally dog-bone shaped with narrow regions $3 \mathrm{~mm}$ in length. The maximum average linear strain rate for these samples would therefore be $4.4 \% / \mathrm{sec}$. Reduction gear boxes with different gear ratios are readily available. Replacing the existing gear box with one having a gear ratio of $32: 1$ would allow the maximum strain rate to be increased to $17.6 \% / \mathrm{sec}$. Smaller gear ratios would produce even higher strain rates but the corresponding reduction in the maximum applied force would limit the stage to low tensile strength samples.

To minimize non-axial stresses on the sample and guide rods, the shaft is connected to the left sample clamp by a two-point pivot assembly. Since the connections on the pivot have a great deal of play, the pivot also reduces the introduction of undesirable rotations around the shaft axis. At a later date, this pivot assembly may be replaced by a rotating point contact to reduce these rotations even further.

Since non-axial forces on the sample must be avoided, the two sample clamps are held in alignment by two hardened steel guide rods. Each guide rod has one threaded end that screws into the right-hand clamp. The smooth rods slide through close-fitting holes that extend completely through the left-hand sample clamp.

Proper design of the sample clamping mechanism is critical if sensitive single-crystal samples are used. Figure 3 shows an exploded view of one of the sample clamps. The sample is held between the base and the lower clamp. Four guide screws fit into the untapped outermost four holes in the two clamps (marked by dotted lines) and screw into the base. These screws are left slightly loose and do not clamp the mechanism. The two clamping screws are inserted into the two threaded holes in the middle of the upper clamp (marked by dashed lines). Tightening these screws presses the lower clamp against the sample. Since force is applied to the lower clamp at just two point contacts aligned along the tensile axis, this design distributes the force equally on both sides of the sample. Since sharp edges can damage a sample, the sample-contacting edges of the base and lower clamp are smoothly 
rounded (not shown in figure).

During a tensile experiment, the right sample clamp (see Fig. 2) is pulled by the sample against two identical rubber compression springs. The displacement of the right clamp is measured by two small capacitance sensors mounted on position-adjustable bases. These sensors have a rated resolution of $0.25 \mu \mathrm{m}$ and a linear range of $2.5 \mathrm{~mm}$. The force versus displacement curves for the springs are calibrated using known weights. The small capacitance sensors can then be used to measure the total tensile force on the sample during an experiment. The average longitudinal stress is calculated by dividing the measured force by the sample's initial cross-sectional area.

The displacement of the left sample clamp is measured using two large capacitance sensors mounted directly on the left side of the frame. These sensors have a resolution of $0.6 \mu \mathrm{m}$ and a linear range of $6.4 \mathrm{~mm}$. Combining the measurements of the large and small capacitance sensors gives not only the total elongation of the sample (assuming compliance in the clamps is negligible), but also a direct measurement of any in-plane rotations experienced by the sample. The measured elongation allows us to calculate the average linear strain, $\epsilon=\Delta L / L_{0}$, where $L_{0}$ is the initial sample length and $\Delta L$ is the measured elongation.

The frame attachment points for the sample clamps are adjustable to allow a range of sample sizes. The maximum possible distance between the sample clamps is $13 \mathrm{~mm}$. Any sample whose final length between the clamps is smaller than this can be accommodated by the tensile stage. To prevent damage to the tensile stage during operation, a limit switch (not shown in figure) was included that automatically stops the stepping motor if the limit of travel is reached.

Since X-ray measurements must be made on these samples, a low profile is highly desirable. The angular acceptances are described by angles measured with respect to a line centered on and perpendicular to the sample. "Top angles" refer to angular acceptances on the side of the stage shown in Fig. 2. The top and bottom acceptance angles in the plane perpendicular to the tensile axis are $69.4^{\circ}$ and $76^{\circ}$, respectively. Thus, the sample stage subtends only $34.6^{\circ}$ on each side of the sample. For angles measured parallel to the tensile axis (along the stage length), shadowing is due to the sample clamps and therefore varies according to the clamp positions. For our normal initial clamp separation of $8 \mathrm{~mm}$, the top and bottom angular acceptances are $56^{\circ}$ and $40^{\circ}$, respectively. The large angular acceptances of this tensile stage allow a great deal of flexibility when planning an X-ray experiment.

\section{SYSTEM PERFORMANCE}

Since the average longitudinal stress is calculated using a measured displacement against a spring system, the spring constant must be measured. This was measured by mounting the tensile stage with the tensile axis vertical, removing the worm gear assembly and hanging weights from the right sample clamp. Figure 4 shows a typical calibration curve for the springs normally used in the tensile stage. Linear regression of the loading curve yields a spring constant of $3.128 \times 10^{-2} \mathrm{~V} / \mathrm{N}$. This system, therefore, has a maximum force resolution of $0.03 \mathrm{~N}$. The small hysteresis and non-linearity are acceptable for the ongoing X-ray studies. For applications where these effects are a problem, sub-miniature load sensors can easily be 
substituted for the springs. The existing springs, however, have an important advantage over conventional load sensors; when the motor is halted for an X-ray measurement, the springs more effectively maintain the load on the sample.

Figure 5 shows an engineering stress-strain curve measured by this system. The noise in this curve is not inherent in the system; it was produced by a noisy $A / D$ board that will be replaced. The dog-bone shaped sample was made from commercial cold-rolled aluminum (polycrystalline) with a thickness of $0.127 \mathrm{~mm}$ and a width of $3 \mathrm{~mm}$; the length of the narrow region was $3 \mathrm{~mm}$. The average linear strain was calculated from the measured sample elongation as described above. The sample was pulled at a constant velocity of 1 $\mu \mathrm{m} / \mathrm{s}$ which corresponds to a strain rate of $0.03 \% / \mathrm{sec}$. The yield and ultimate strength, as well as the shape of the resulting curve, are typical of aluminum.

The tensile stage system has been used on beam line X23A3 at the NSLS for in situ highresolution diffraction imaging of strained single-crystal samples of $\mathrm{Al}^{7}{ }^{7}$ In one experiment, the strain was applied monotonically along an axis within $2^{\circ}$ of $(33 \overline{1})$. Stereopair images from a single crystal were taken in situ at strain values of $0 \%, 0.45 \%, 1.06 \%, 1.94 \%, 2.74 \%$ and $3.65 \%$. Individual images were then taken at strains of $4.52 \%, 5.4 \%, 7.20 \%$ and $9.00 \%$. Figure 6 shows transmission diffraction images of this sample taken using the $020 \mathrm{Bragg}$ peak. In this figure, white regions signify dislocations and dislocation structures. The grainyness is due to the low resolution of the film; Future work will use high resolution nuclear emulsions with a grain size of 0.1 micrometers.

Figure 6(a) shows the sample before deformation. The sharp white features are dislocation braids meandering through the sample. The full path of the braids in three dimensions can be determined from the stereopair image data. Faint, broadened lines are also visible extending down and to the right in Fig. 6. These are individual dislocations comprising a very small-angle tilt boundary situated toward the X-ray entrance side of the crystal; the dislocations have an average separation of 100 micrometers. Due to the image formation process, features are much sharper for structures located near the X-ray exit surface of the crystal.

Figure 6(b) shows a 020 transmission diffraction image from the same region of the same sample after applying an average linear strain of $0.45 \%$. The most pronounced change is the large movement of the braids. The braids have also become significantly thinner in this image. In future experiments, we will use a smaller strain increment to follow these changes in detail. Finally, the dislocations in the small angle tilt boundary have moved closer to the exit surface of the crystal, thus demonstrating migration of this boundary.

In situ SAXS experiments were also conducted on a strained single-crystal Al sample. ${ }^{8} \mathrm{As}$ with the diffraction imaging sample described above, the sample was strained monotonically along a (33ī) axis. Data were taken at strain values of $0 \%, 0.47 \%, 1.28 \%, 2.5 \%, 5.0 \%$, $8.33 \%$ and $16.67 \%$. The tensile-stage system performed flawlessly throughout all of the $\mathrm{X}$-ray experiments.

\section{CONCLUSION}

We have designed, built and tested a remotely controlled, low-profile, light-weight tensile stage system that can uniaxially deform small samples in situ while mounted on an X- 
ray goniometer. Applied force and relative displacement measurements are made at $0.5 \mathrm{~s}$ intervals throughout the experiment and are stored on the controlling computer system. These data are used to calculate the average longitudinal stress and the average linear strain. The calculated strain is also displayed in real time on the computer. In situ tensile experiments on single-crystal Al samples at the NSLS confirm that the new tensile stage system can be used effectively for X-ray studies of deformation.

Although this system was primarily designed for monotonic sample deformation, other experiments are also possible. Creep can be studied by straining a sample and leaving it under a constant stress while taking X-ray data. Furthermore, introduction of a software feedback loop would allow samples to be cyclicly fatigued by repeatedly loading and unloading the sample. 


\section{REFERENCES}

${ }^{1}$ Results of U.S. government work not subject to copyright.

${ }^{2}$ Kyoichi Haruta, J. Appl. Phys., 76, 1789 (1965)

${ }^{3}$ B. Steiner, L. E. Levine, M. Brown and D. Larson, accepted by J. Cryst. Growth, (1996)

${ }^{4}$ L. E. Levine and Robb Thomson, unpublished (1996)

${ }^{5}$ T. Ungár, H. Mughrabi, D. Rönnpagel, and M. Wilkens, Acta metall., 32, 333 (1984)

${ }^{6}$ H. Mughrabi, T. Ungár, W. Kienle, and M. Wilkens, Phil. Mag., A53, 793 (1986).

${ }^{7}$ L. E. Levine, D. R. Black and G. Long, unpublished (1996)

${ }^{8}$ L. E. Levine and G. Long, unpublished (1996) 


\section{FIGURES}

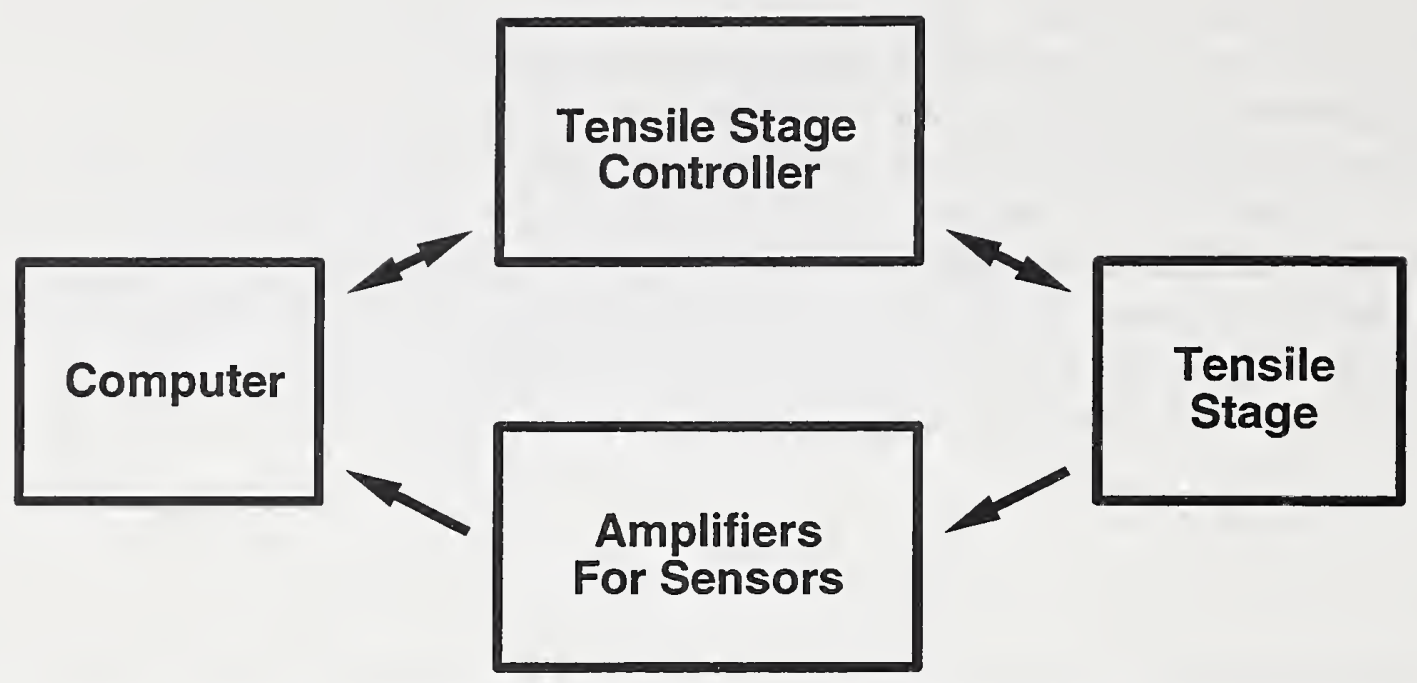

FIG. 1. Block diagram of tensile stage system. The arrows indicate the communication paths.

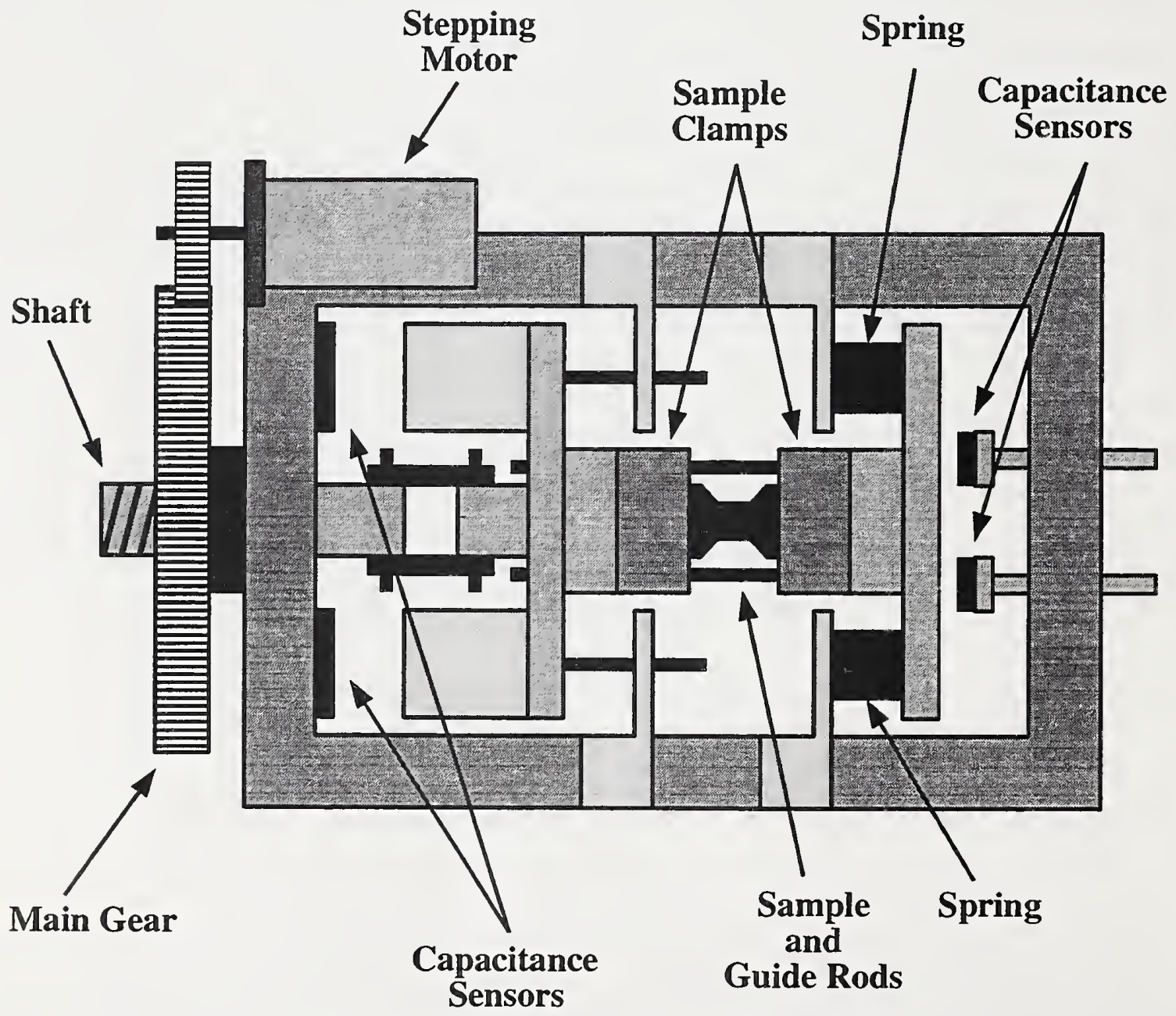

FIG. 2. Detailed diagram of tensile stage. 


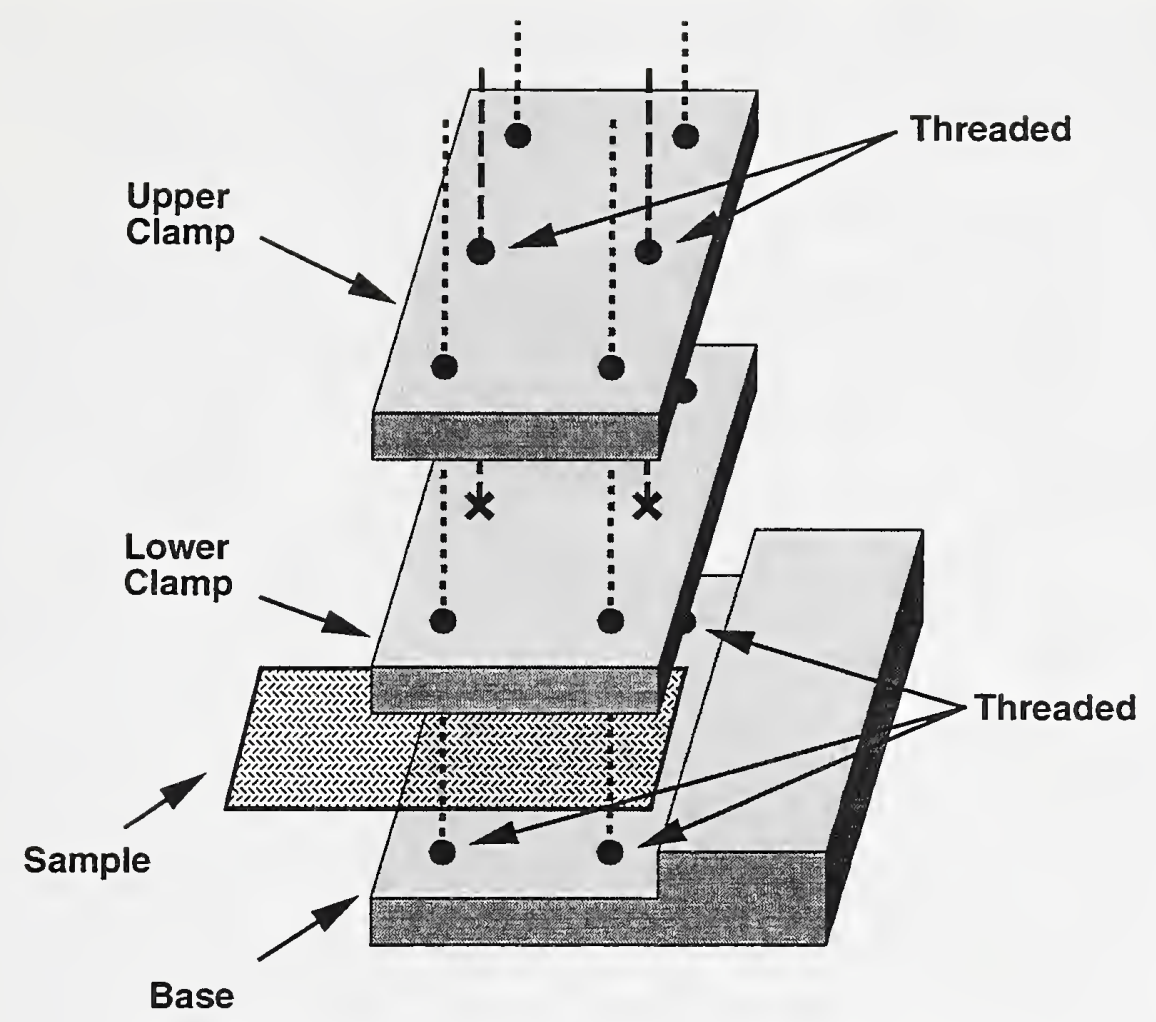

FIG. 3. Exploded view of sample clamp mechanism.

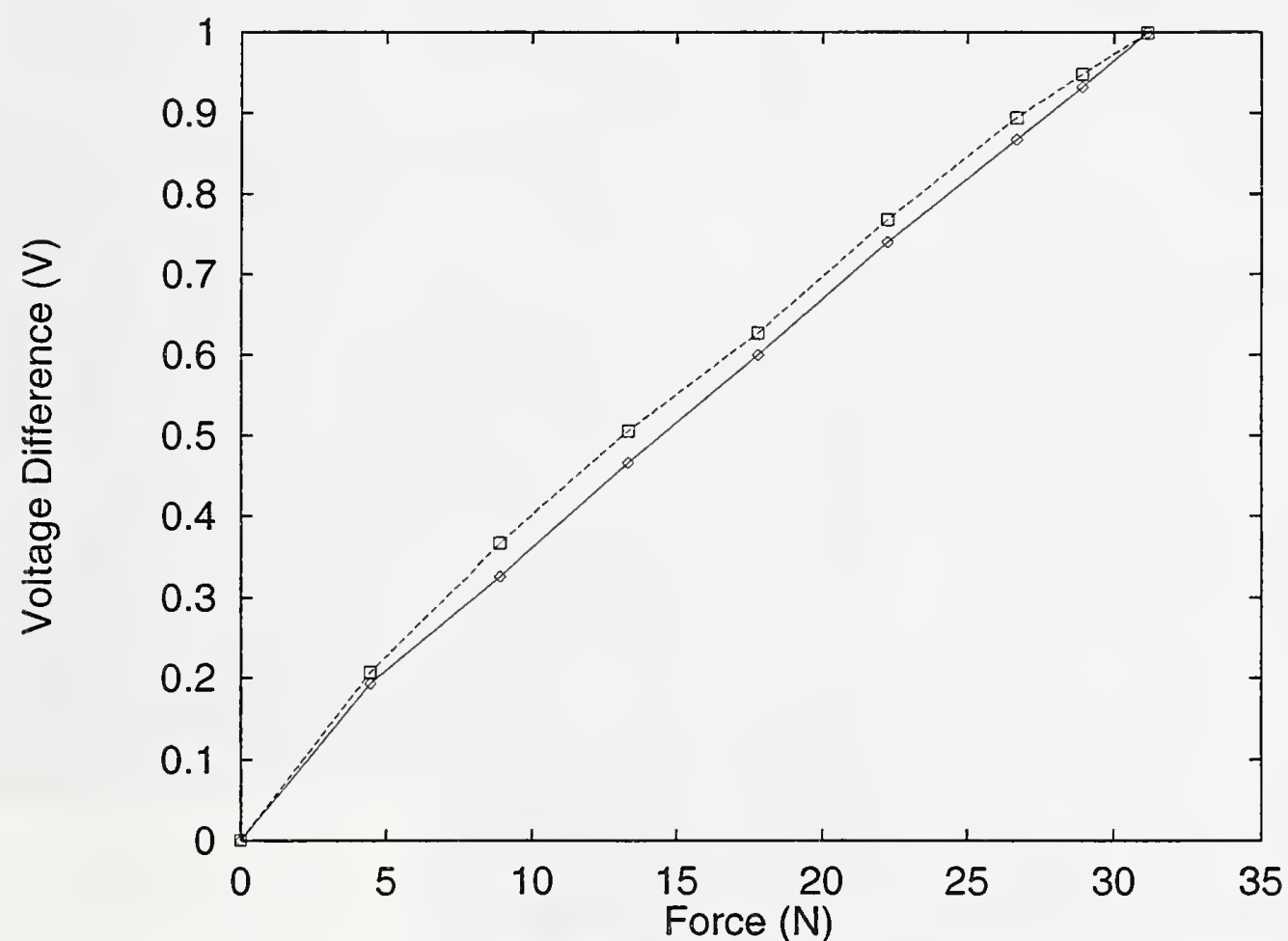

FIG. 4. Calibration curves for rubber springs. The solid and dashed lines represent loading and unloading, respectively. 


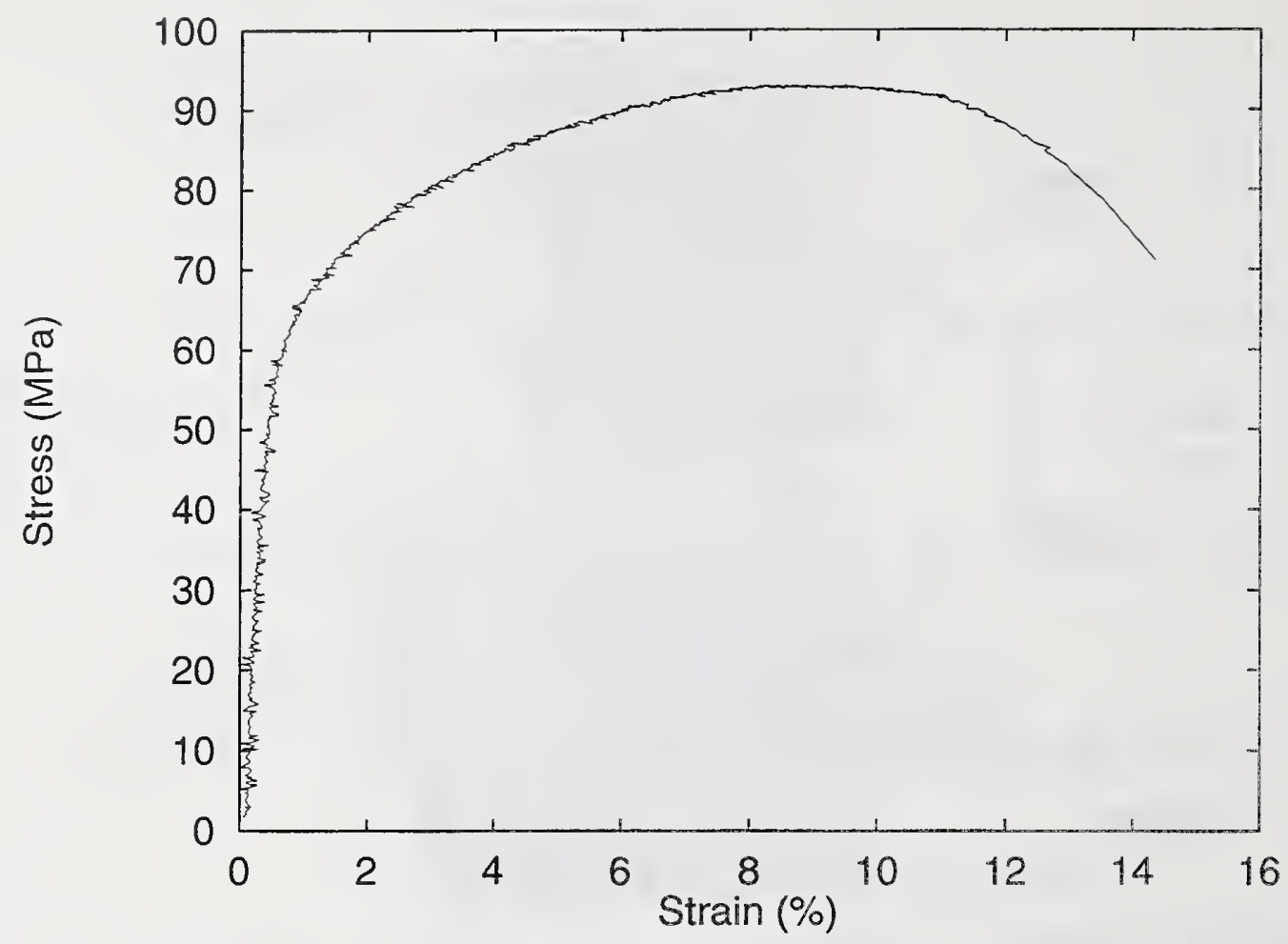

FIG. 5. Engineering stress-strain curve for cold-rolled polycrystalline AI.

FIG. 6. High-resolution 020 X-ray diffraction images from a single-crystal Al sample strained uniaxially along the $(33 \overline{1})$ axis in situ. (a) unstrained sample (b) sample strained $0.45 \%$ 


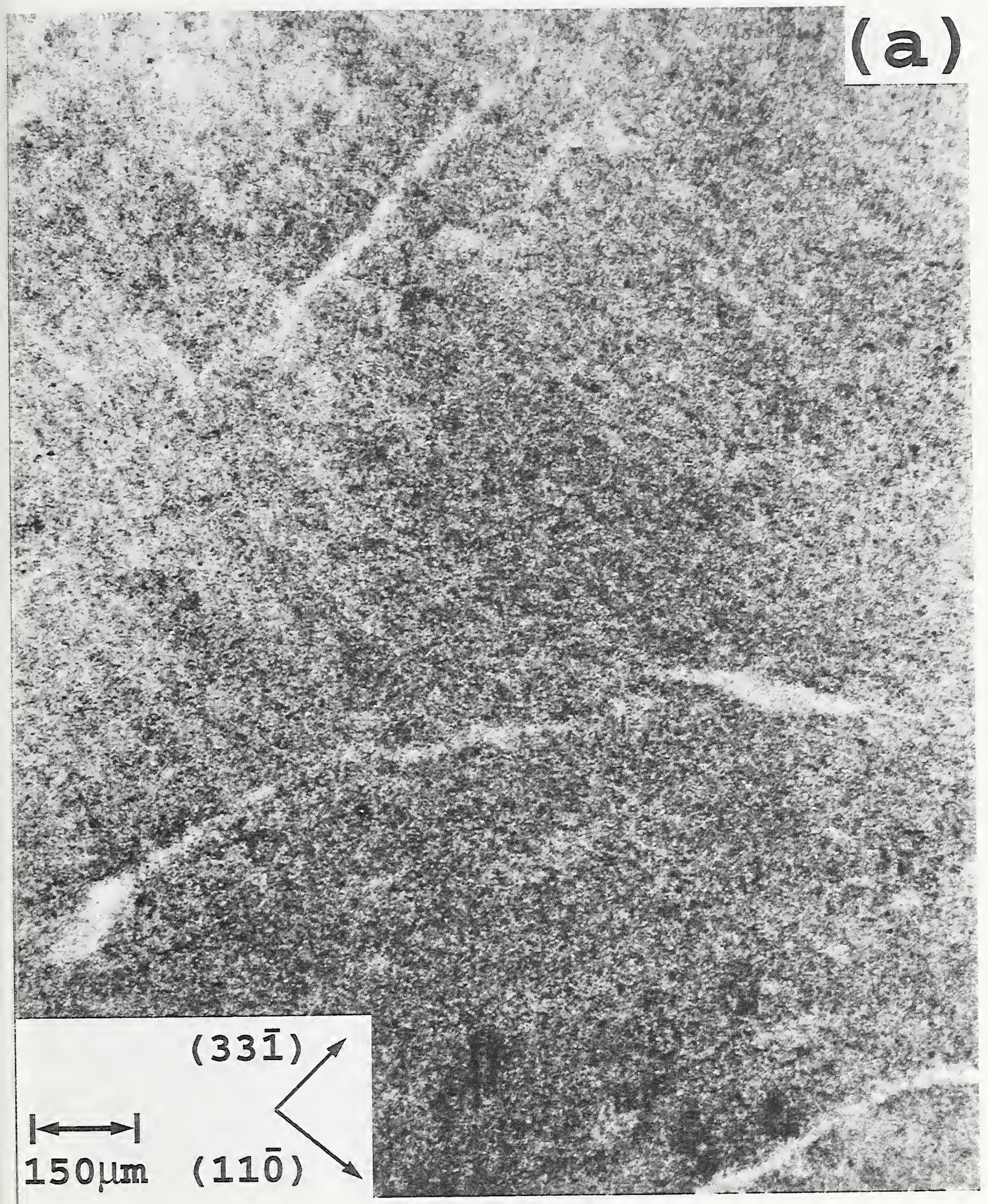



$4 y-17$

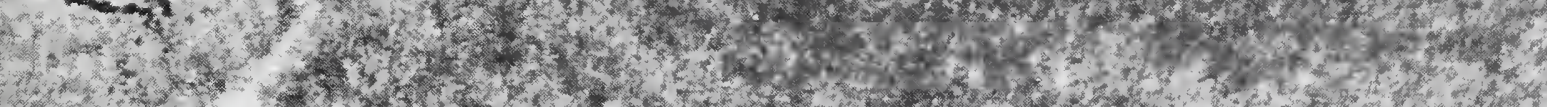

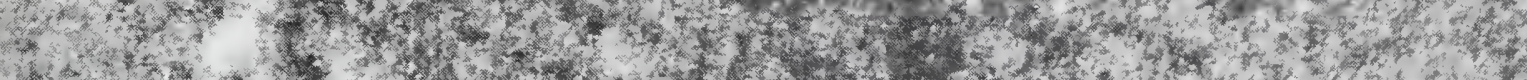

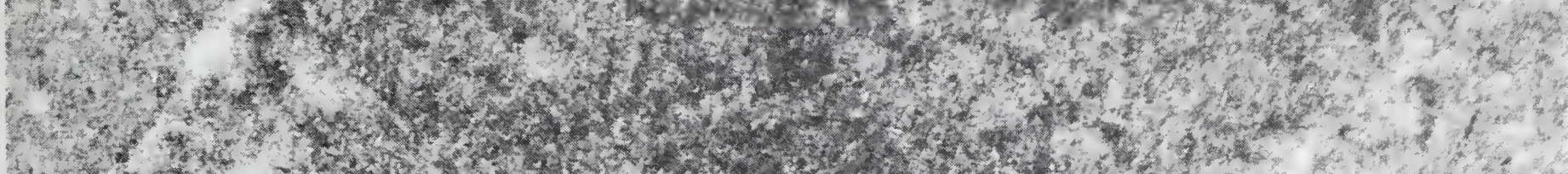

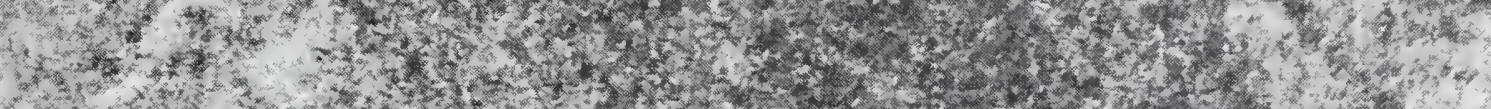

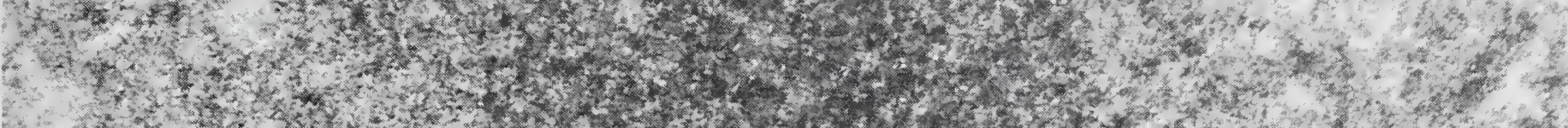

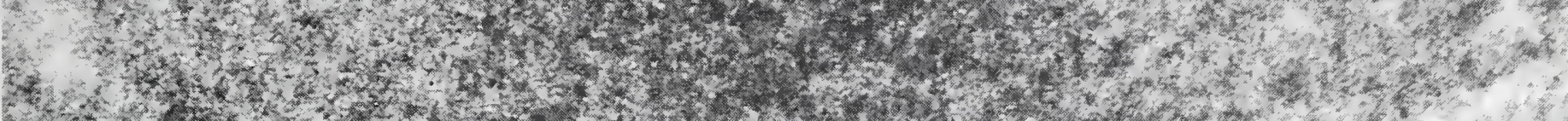

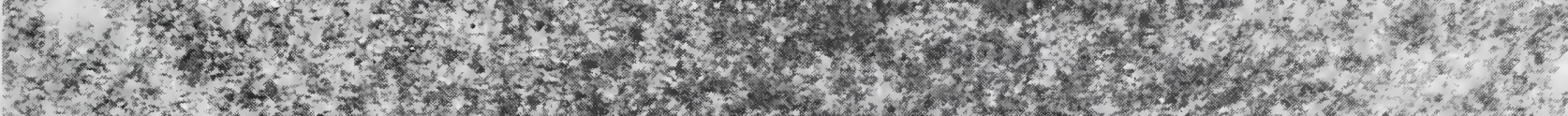

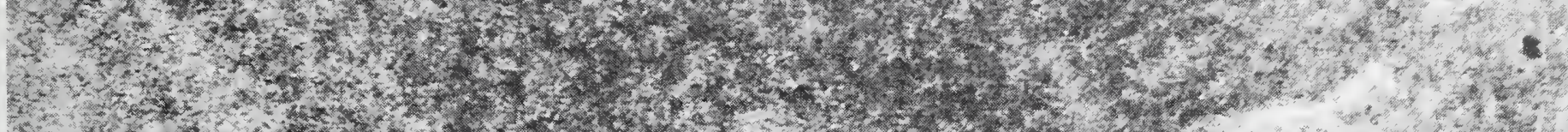

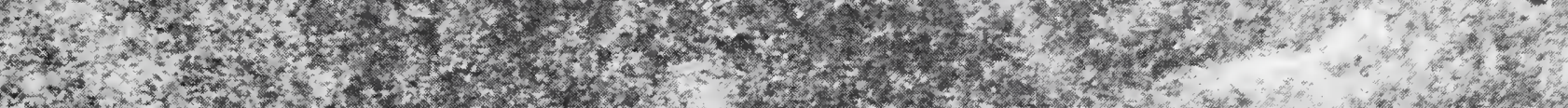

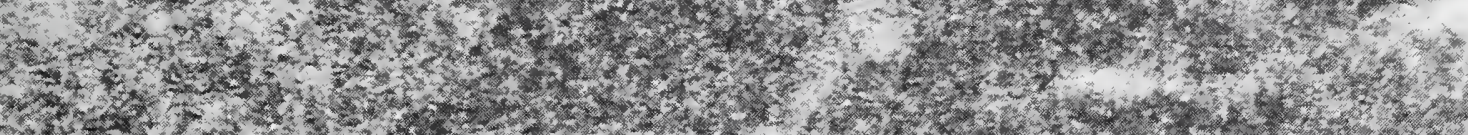

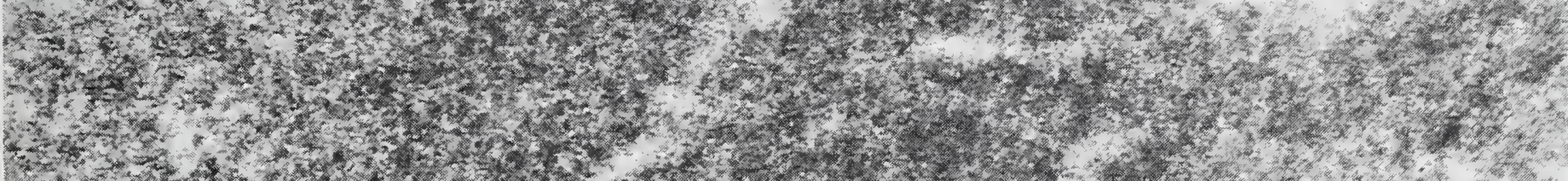

1
1

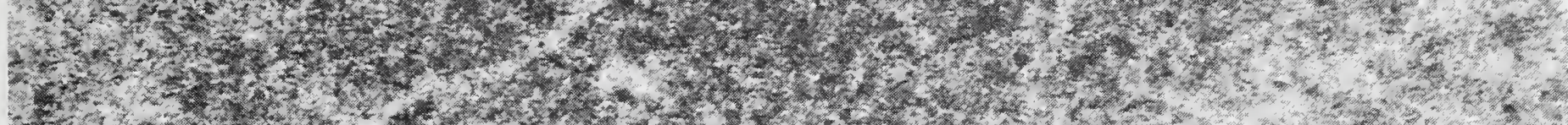

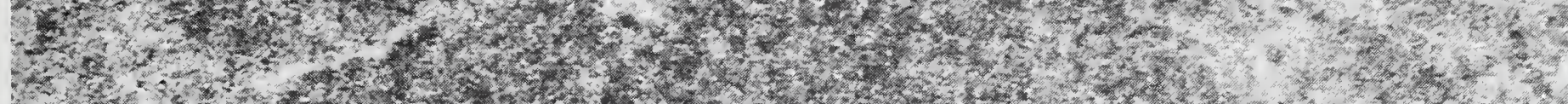

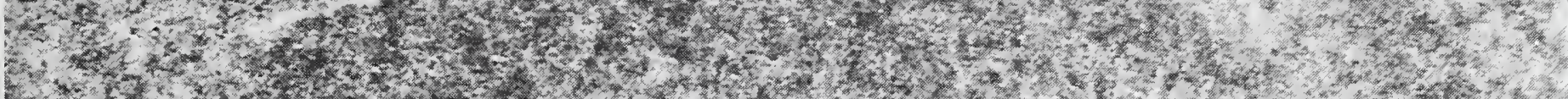

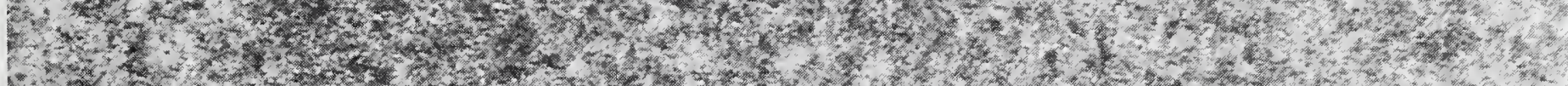

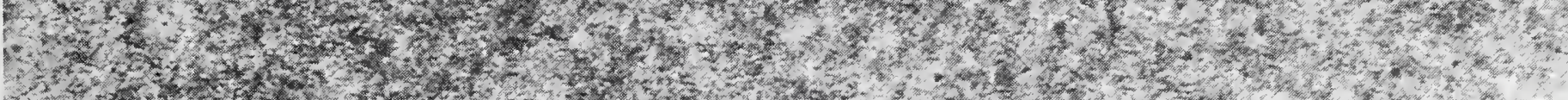

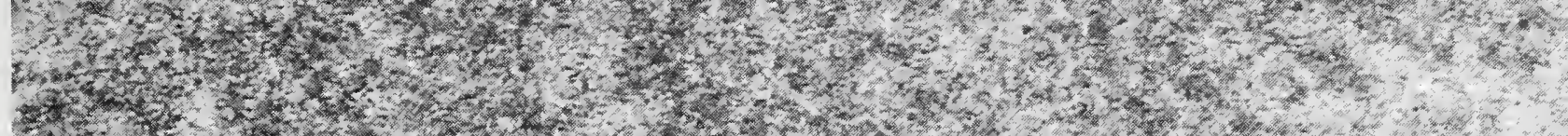

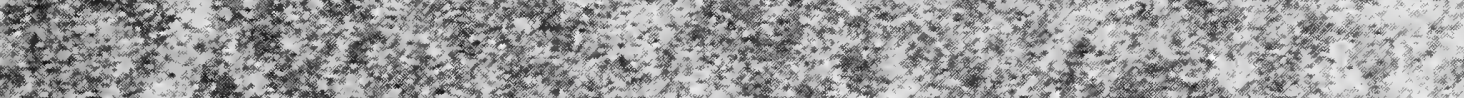

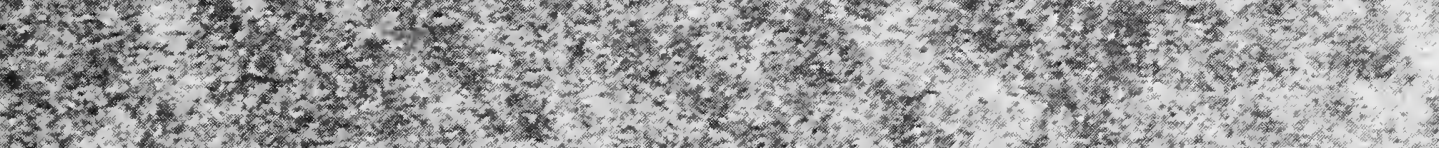

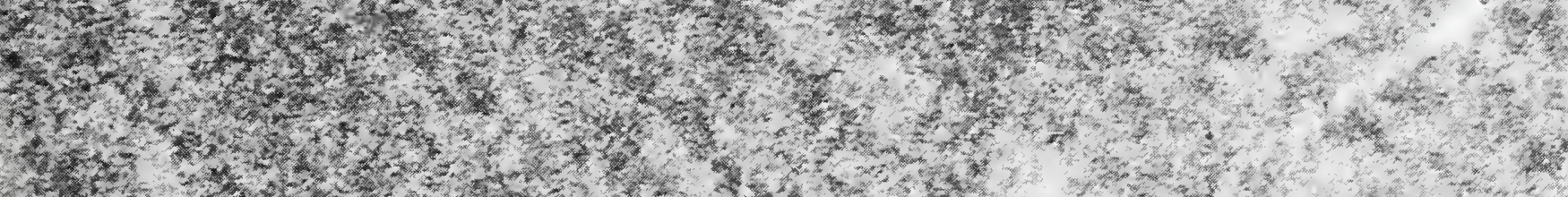

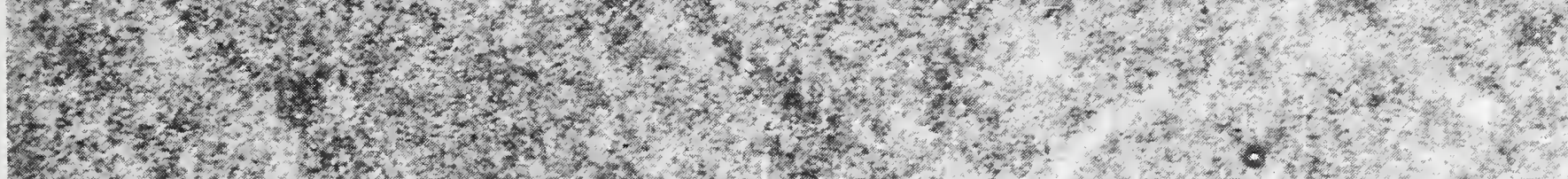

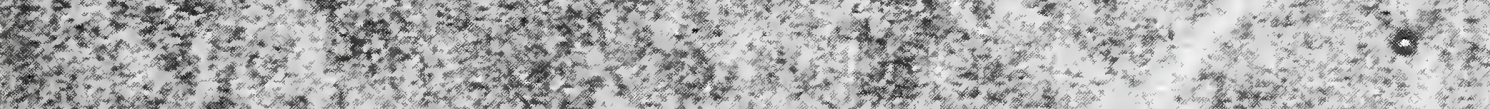

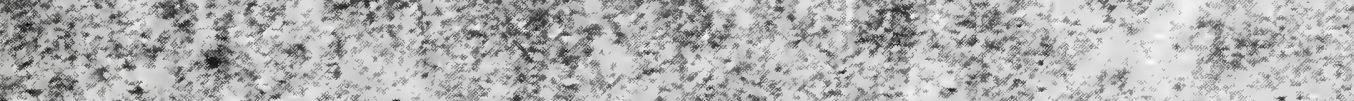

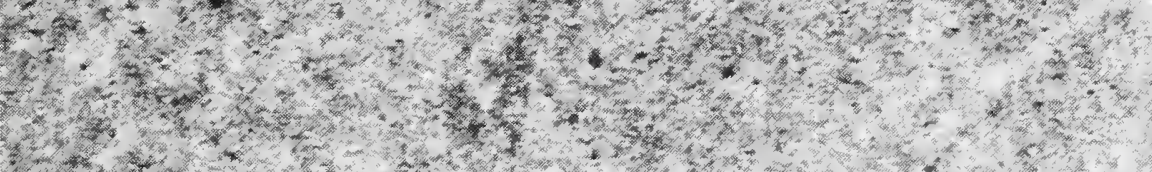

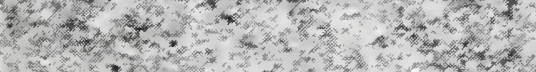

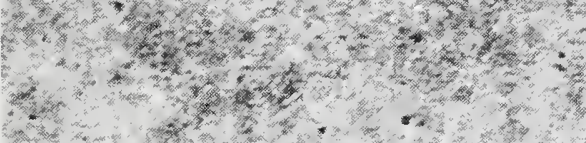




